\title{
USING SPINNING DUST EMISSION TO CONSTRAIN THE ABUNDANCE OF VERY SMALL DUST GRAINS IN DENSE CORES
}

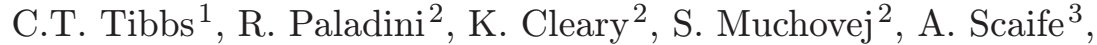 \\ M. Stevenson ${ }^{2}$, R. Laureijs ${ }^{1}$, N. Ysard ${ }^{4}$, K. Grainge ${ }^{3}$, Y. Perrott ${ }^{5}$, \\ C. Rumsey ${ }^{5}$ and J. Villadsen ${ }^{2}$
}

\begin{abstract}
We present the first analysis of using spinning dust emission as a method to characterise the properties of very small interstellar dust grains in dense cores.
\end{abstract}

\section{Introduction}

Within many molecular clouds in our Galaxy there are cold dense structures known as pre-stellar cores. These cores represent one of the earliest phases of star formation and they also play a vital role in the life cycle of dust in the interstellar medium, making them an ideal location in which to study dust grain evolution. To date, all of the studies to constrain dust grain properties have come from observations at ultraviolet, optical, and infrared wavelengths, but the goal of this work is to characterise the properties of interstellar dust grains using observations at $\mathrm{cm}$ wavelengths from spinning dust emission. Spinning dust emission, produced by electric dipole radiation from very small spinning dust grains (Draine \& Lazarian 1998), has been observed at $\mathrm{cm}$ wavelengths in a range of Galactic environments (e.g., Tibbs et al. 2010, 2012). Recent modelling by Ysard et al. (2011) shows that if very small dust grains are present in dense cores, they will produce spinning dust emission, and therefore spinning dust emission can be used to determine the presence, and abundance, of very small dust grains in dense cores.

\footnotetext{
${ }^{1}$ ESA/ESTEC, Noordwijk, 2201 AZ, The Netherlands; e-mail: ctibbs@cosmos.esa.int

${ }^{2}$ California Institute of Technology, Pasadena, CA 91125, USA

${ }^{3}$ University of Manchester, Manchester, M13 9PL, UK

${ }^{4}$ Université Paris-Sud, 91405 Orsay Cedex, France

${ }^{5}$ University of Cambridge, Cambridge, CB3 0HE, UK
} 

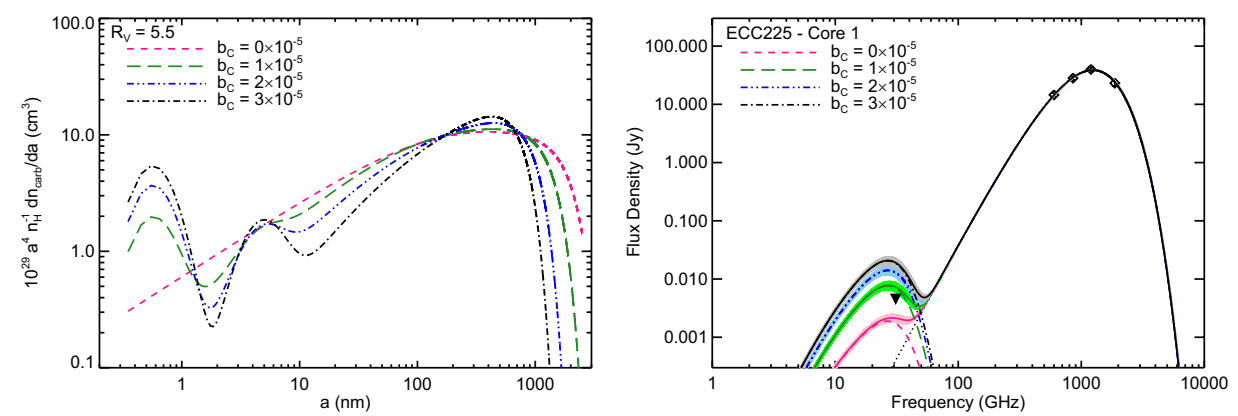

Fig. 1. Left: dust grain size distributions (Tibbs et al. 2015). Right: SED of one of our cores (Tibbs et al. 2016). The spinning dust curves are produced using SPDUST for four different very small dust grain abundances $\left(b_{\mathrm{C}}\right)$, while the $1 \mathrm{~cm}$ data point (solid triangle) is the $5 \sigma$ upper limit estimated from the CARMA data. Also plotted are the flux densities of the Herschel data (diamonds) at 160, 250,350, and $500 \mu \mathrm{m}$.

\section{Constraining the abundance of very small dust grains}

Tibbs et al. (2015) observed a sample of dense cores at a wavelength of $1 \mathrm{~cm}$ using the CARMA interferometer to search for spinning dust emission. Additionally, they used Herschel observations to estimate the mean density and radiation field for each core. With these physical properties of the cores, we use a spinning dust model (SPDUST; Silsbee et al. 2011) to estimate the expected level of spinning dust emission for four dust grain size distributions. By comparing the predicted levels of spinning dust emission to the observed CARMA measurements, we can constrain the abundance of very small dust grains, $b_{\mathrm{C}}$. In Figure 1 we plot the dust grain size distributions and the SED for one of our cores, illustrating how spinning dust emission can constrain the abundance of very small dust grains in dense cores.

\section{Conclusion}

This work represents the first attempt at using spinning dust emission to characterise the properties of interstellar dust grains, and we illustrate this by using spinning dust to constrain the abundance of very small dust grains in dense cores.

\section{References}

Draine, B.T., \& Lazarian, A., 1998, ApJ, 508, 157

Silsbee, K., Ali-Haïmoud, Y., \& Hirata, C.M., 2011, MNRAS, 411, 2750

Tibbs, C.T., Watson, R.A., Dickinson, C., et al., 2010, MNRAS, 402, 1969 
Tibbs, C.T., Paladini, R., Compiegne, M., et al., 2012, ApJ, 754, 94

Tibbs, C.T., Paladini, R., Cleary, K., et al., 2015, MNRAS, 453, 3356

Tibbs, C.T., Paladini, R., Cleary, K., et al., 2016, MNRAS, 456, 2290

Ysard, N., Juvela, M., \& Verstraete, L., 2011, A\&A, 535, A89 